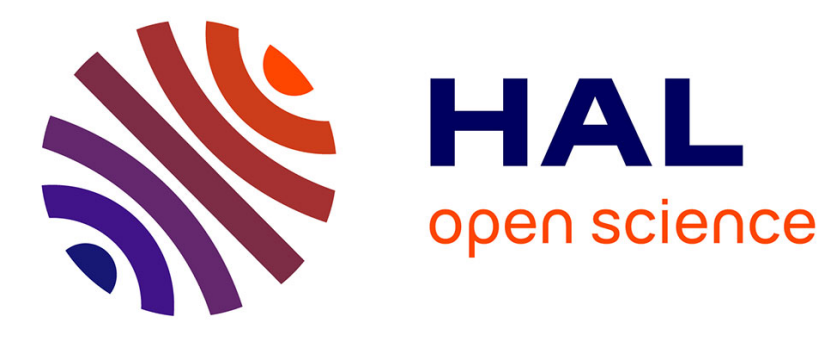

\title{
An Optimistic Planning Approach for the Aircraft Landing Problem
}

Sana Ikli, Catherine Mancel, Marcel Mongeau, Xavier Olive, Emmanuel Rachelson

\section{- To cite this version:}

Sana Ikli, Catherine Mancel, Marcel Mongeau, Xavier Olive, Emmanuel Rachelson. An Optimistic Planning Approach for the Aircraft Landing Problem. EIWAC 2019:, 6th ENRI International Workshop on ATM/CNS, ENRI, Oct 2019, Tokyo, Japan. pp 173-188, 10.1007/978-981-33-4669-7_10 . hal-02382692

\section{HAL Id: hal-02382692 \\ https://hal-enac.archives-ouvertes.fr/hal-02382692}

Submitted on 27 Nov 2019

HAL is a multi-disciplinary open access archive for the deposit and dissemination of scientific research documents, whether they are published or not. The documents may come from teaching and research institutions in France or abroad, or from public or private research centers.
L'archive ouverte pluridisciplinaire HAL, est destinée au dépôt et à la diffusion de documents scientifiques de niveau recherche, publiés ou non, émanant des établissements d'enseignement et de recherche français ou étrangers, des laboratoires publics ou privés. 


\title{
[EN-A-25] An Optimistic Planning Approach for the Aircraft Landing Problem
}

(EIWAC 2019)

\author{
${ }^{+}$S. Ikli*, C. Mancel*, M. Mongeau*, X. Olive**, E. Rachelson*** \\ *ENAC, Université de Toulouse \\ Toulouse, France \\ [sana.ikli I catherine.mancel I mongeau]@ recherche.enac.fr \\ ${ }^{* *}$ ONERA DTIS, Université de Toulouse \\ Toulouse, France \\ xavier.olive@onera.fr \\ **ISAE-SUPAERO, Université de Toulouse \\ Toulouse, France \\ emmanuel.rachelson@isae-supaero.fr
}

\begin{abstract}
:
The Aircraft Landing Problem consists in sequencing aircraft on the available runways and scheduling their landing times taking into consideration several operational constraints, in order to increase the runway capacity and/or to reduce delays. In this work we propose a new Mixed Integer Programming (MIP) model for sequencing and scheduling aircraft landings on a single or multiple independent runways incorporating safety constraints by means of separation between aircraft at runways threshold. Due to the NP-hardness of the problem, solving directly the MIP model for large realistic instances yields redhibitory computation times. Therefore, we introduce a novel heuristic search methodology based on Optimistic Planning that significantly improve the FCFS (First-Come First-Served) solution, and provides good-quality solutions in reasonable computational time. The solution approach is then tested on medium and large realistic instances generated from real-world traffic on Paris-Orly airport to show the benefit of our approach.
\end{abstract}

Keywords: Aircraft Landing problem, Mixed Integer Programming, Optimistic Planning

\section{INTRODUCTION}

The International Air Transport Association (IATA) expects 7.8 billion passengers to travel in 2036, which represents nearly double the passengers recorded in 2016 [1]; this increasing demand on air transportation exposes the available infrastructure to a risk of saturation. Constructing new infrastructures (runways, airports) is a solution to increase the capacity, however, it may not always be feasible due to the huge cost incurred. The alternative is to optimize the use of current infrastructure, especially the runway which is recognized to be the bottleneck of the whole Air Traffic Management (ATM) system.

Since the runway sequence is one of the key factors that determines runway capacity [2], several researchers were interested in the optimization of runway sequences, which corresponds in the literature to the following problems:

- The Aircraft Landing Problem (ALP) aims at sequencing arriving aircraft on the available runways and scheduling their landing times taking into consideration several operational constraints.

- The Aircraft Take-off Problem (ATP) consists in scheduling take-off slots to departing aircraft
- The Aircraft Scheduling Problem (ASP) consists in sequencing and scheduling simultaneously departing and arriving aircraft.

According to the survey [3] by Bennell et al., the ALP received much more attention in the literature than the ATP or the ASP. Several approaches are proposed in the literature for the three above-mentioned problems, and can be classified in two main categories:

- Exact approaches, mainly MIP-based approaches ([4], [5], [6], [7], [8], [9]) and Dynamic Programming ([10], [6], [11])

- Heuristic approaches ([12], [4], [9]) and Metaheuristics, such as Simulated Annealing ([13], [14], [15], [16]), Tabu Search ([8], [17]), Genetic Algorithms ([18], [19]), Ant Colony Optimization ([20], [21]), and Variable Neighborhood Search ([22], [13]).

Interested readers may refer to [3] for a comprehensive review of existing approaches to the ALP.

In this work, we are interested in sequencing and scheduling aircraft landings at the runway threshold. Each aircraft has a target landing time and an authorized landing time window, expressed as an earliest and a latest acceptable landing 
time based on fuel considerations. Deviations from the target times will cause a cost that depends on each aircraft, and the aim is to minimize the total deviations from target times, which is more general than minimizing only total schedule tardiness. To model the problem, we propose a novel MIP formulation that takes into consideration safety constraints by imposing separation between aircraft at the runway threshold (Table 1). The proposed formulation is adapted to airports that involve multiple independent runways. Due the the NP-hardness of the problem [4], solving directly the MIP model for large realistic instances leads to redhibitory computation times, which is unsuited for the dynamic nature of the problem that requires air-traffic controllers to make quick but good decisions. Therefore, we introduce a novel heuristic search methodology based on Optimistic Planning [23], that provides good-quality solutions in a negligible time. We then evaluate empirically our approach on realistic instances generated from real-traffic data from Paris-Orly airport.

The remainder of this paper is organized as follows. In Section 2 we describe the ALP and highlight the operational constraints. Next, Section 3 presents our proposed MIP formulation and the constraints taken into account. Then, in Section 4, we explain our proposed solution approach. Section 5 presents computational results that show the benefits of our approach, and finally in Section 6 we summarize the contributions of this work and suggest some perspectives for future research work.

\section{PROBLEM DESCRIPTION}

Given a set of aircraft near the terminal area of an airport, the ALP consists in mapping each aircraft to a landing time such that a given criterion is optimized while operational constraints are satisfied. When the airport has more than one runway, a decision with respect to the landing runway has to be made by controllers; the runway assignment depends on several factors such as the airport configuration and the direction of arriving aircraft.

The most common approach used by controllers to sequence aircraft is the First-Come First-Served (FCFS) rule, where aircraft land according to the order of the scheduled times of arrival at the runway, and air-traffic controllers ensure only the minimum separation requirements. This FCFS heuristic is easy to implement and guarantees equity between aircraft. However, it is rarely optimal in terms of runway throughput, especially in congested airports [10], simply consider the large separation requirement in some scenarios where a heavy aircraft is followed by a light aircraft (Figure 1). This motivates the development of efficient methods that compute optimal sequences while satisfying several operational constraints such as minimum separation, authorized time windows and constrained-position shifting.

\begin{tabular}{|cc|ccc|}
\hline & & \multicolumn{3}{|c|}{ Following aircraft } \\
& & $\mathrm{H}$ & $\mathrm{M}$ & $\mathrm{L}$ \\
\hline \multirow{3}{*}{ Leading aircraft } & $\mathrm{H}$ & 96 & 157 & 196 \\
& $\mathrm{M}$ & 60 & 69 & 131 \\
& $\mathrm{~L}$ & 60 & 69 & 82 \\
\hline
\end{tabular}

Table 1: Final approach separation matrix (in seconds) according to ICAO's basic wake-turbulence categories (Source [10])

- The minimum separation constraint guarantees that no aircraft is affected by the wake-vortex turbulence generated by a leading aircraft, especially during takeoffs and landings. The International Civil Aviation Organization (ICAO) classifies aircraft in three main categories, namely Heavy (H), Medium (M) and Light (L), and the separation requirements are defined depending on the category of both the leading and the following aircraft. Separation requirements are given in Nautical Miles (NM), but can be converted to seconds as explained in [2] and summarized in Table 1.

- Time-windows constraints are defined by an earliest and a latest possible landing times, based on fuel availability or on possible speed-ups. Indeed, once an aircraft arrives at the boundary of a radar range (about 45-60 minutes from the destination airport), decisionsupport tools compute an Estimated-Time of Arrival (ETA) at the runway threshold. If the aircraft speeds up, the Actual Landing Time (ALT) may be earlier than the ETA. On the other hand, aircraft may by delayed after entering the radar range and, in this case, the ALT will be later that the ETA and the latest possible landing time is limited by the available fuel [24].

- The Constrained-Position Shifting (CPS) constraint limits the deviation from the FCFS sequence for equity reasons. This constraint ensures that an aircraft is not deviated from its initial position in the FCFS sequence by more than a given number of positions called maximum position shifting and denoted $m$, which is usually small; $m=3$ or 4 [10]. This constraint does not only ensure equity between aircraft, but it also reduces the complexity of the problem.

In the following section, we introduce a MIP formulation for the ALP involving one or multiple runways, and we show how we can incorporate different operational constraints in the model.

\section{MATHEMATICAL MODELING}

Runway assignment and scheduling aircraft at each runway is formulated as a MIP model which decides the landing dates at each runway threshold, while respecting safety requirements so as to optimize a given objective. We leave the control problem, i.e. how aircraft can be controlled so as to implement the solution of our decision problem, for future research work. 


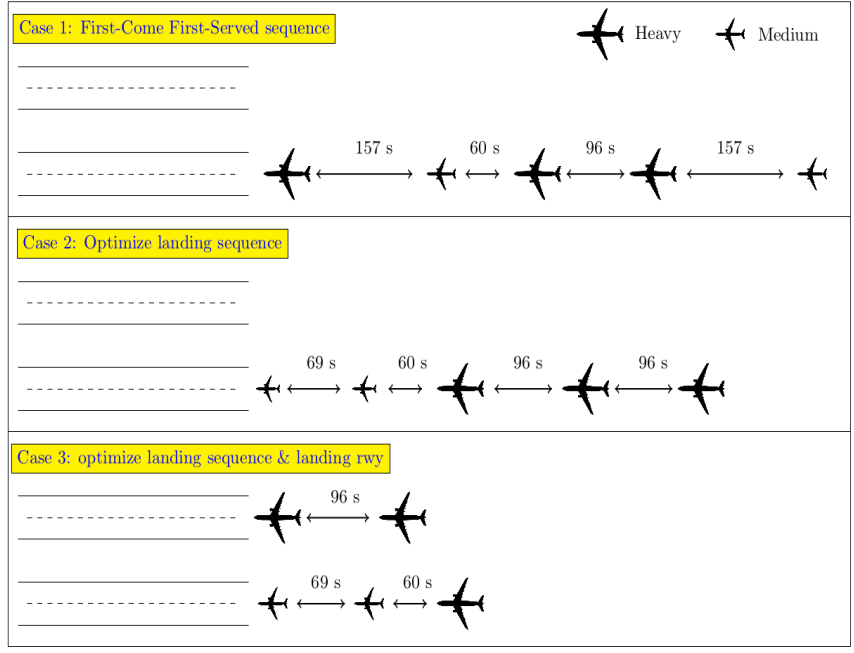

Figure 1: Comparison of three landing sequences. Case 1 illustrates the FCFS sequence. In case 2, the landing sequence is optimized with respect to wake turbulence separation, and in case 3 the landing sequence is further optimized by runway assignment.

\begin{tabular}{|c|l|}
\hline Notation & Parameter \\
\hline$T_{i}$ & Target landing time \\
{$\left[E_{i}, L_{i}\right]$} & Landing time window $\left(L_{i}>E_{i}\right)$ \\
$S_{i j}$ & minimum separation time $(\geq 0)$ between aircraft $i$ and \\
$c_{i}^{-}$ & $j$, where $i$ lands before $j$ \\
$c_{i}^{+}$ & $\begin{array}{l}\text { Penalty cost }(\geq 0) \text { per time-unit for landing before the } \\
\text { target time } T_{i} \\
\text { Penalty cost }(\geq 0) \text { per time-unit for landing after the } \\
\text { target time } T_{i}\end{array}$ \\
\hline
\end{tabular}

Table 2: List of input data

\subsection{Input data}

Consider a set of arriving aircraft $\mathcal{A}=\{1,2, \ldots, N\}$, and a set of available runways $\mathcal{K}=\{1,2, \ldots, R\}$. Without loss of generality, let us assume that each aircraft index $i \in \mathcal{A}$ represents its position in the FCFS sequence. Then, for each flight $i \in \mathcal{A}$, the given input data are presented in Table 2 .

\subsection{Decision variables}

Our proposed model involves binary optimization variables for sequencing and runway assignment, and continuous optimization variables for assigning times at the runway threshold. The binary variables are defined as follows:

- $a_{i k}= \begin{cases}1 & \text { if aircraft } i \text { is assigned to runway } k, \\ 0 & \text { otherwise, }\end{cases}$

- $\delta_{i j k}=\left\{\begin{array}{l}1 \text { if aircraft } i \text { and } j \text { are assigned to } \\ \text { runway } k, \text { and } i \text { lands before } j, \\ 0 \text { otherwise, }\end{array}\right.$

- $y_{i j}=\left\{\begin{array}{l}1 \text { if aircraft } i \text { lands before } j \\ 0 \text { otherwise }\end{array}\right.$
For each aircraft $i \in \mathcal{A}$, the continuous variables are:

- $t_{i} \quad$ : landing time

- $t_{i}^{-}, t_{i}^{+}:$deviations from the target landing time $T_{i}$ (before and after $T_{i}$, respectively).

\subsection{MIP model}

Our objective minimizes the total deviation cost from target times $\left(T_{i}\right)$ which is more general than minimizing only the total schedule delay. The complete model is given by (1)-(12)

$$
\begin{aligned}
& \min _{\delta, y, a, t} \sum_{i \in \mathcal{A}} c^{-} \overbrace{\max \left(0, T_{i}-t_{i}\right)}^{t_{i}^{-}}+c^{+} \overbrace{\max \left(0, t_{i}-T_{i}\right)}^{t_{i}^{+}} \\
& t_{i}=T_{i}-t_{i}^{-}+t_{i}^{+} \quad i \in \mathcal{A} \\
& E_{i} \leq t_{i} \leq L_{i} \quad i \in \mathcal{A} \\
& y_{i j}+y_{j i}=1 \quad i, j \in \mathcal{A}: i<j \\
& \sum_{k \in \mathcal{K}} a_{i k}=1 \quad i \in \mathcal{A} \\
& \sum_{k \in \mathcal{K}} \delta_{i j k}+\delta_{j i k} \leq 1 \quad i, j \in \mathcal{A}: i<j \\
& \delta_{i j k}+\delta_{j i k} \geq a_{i k}+a_{j k}-1 \quad i, j \in \mathcal{A}: i<j, k \in \mathcal{K} \\
& 2\left(\delta_{i j k}+\delta_{j i k}\right) \leq a_{i k}+a_{j k} \quad i, j \in \mathcal{A}: i<j, k \in \mathcal{K} \\
& t_{j} \geq t_{i}-M_{1}\left(1-y_{i j}\right) \quad i, j \in \mathcal{A}: i \neq j \\
& t_{j} \geq t_{i}+S_{i j}-M_{2}\left(1-y_{i j}\right) \quad i, j \in \mathcal{A}: i \neq j \\
& i-m \leq N-\sum_{j \in \mathcal{A}, j \neq i} y_{i j} \leq i+m \quad i \in \mathcal{A} \\
& \delta_{i j k}, y_{i j}, a_{i k} \in\{0,1\} \quad i, j \in \mathcal{A}: i \neq j, k \in \mathcal{K}
\end{aligned}
$$

In the above formulation, constraints (2) are introduced to linearize the objective function; constraints (3) represent the time window restrictions; constraints (4) enforce the order precedence relationship between flights $i$ and $j$ at landing; constraints (5) ensure that an aircraft is assigned to exactly one runway; constraints (6) enforce the order precedence relationship between flights landing on the same runway; constraints (7) and (8) enforce the logical relationship between $\delta_{i j k}$ and $a_{i k}$; constraints (9) relates precedence relationships between landings to landing times; constraints (10) ensure the separation requirements between aircraft landing at the same runway; constraints (11) impose the CPS constraint, and constraints (12) enforce the binary restrictions of our discrete variables.

Before reporting numerical results obtained with this formulation, we shall first present a novel alternate methodology to solve the ALP, since solving directly the MIP leads to redhibitory computation times, as we shall show in Section 5. 


\section{OPTIMISTIC PLANNING}

The dynamic nature of the ALP requires air-traffic controllers to make quick but good decisions; the computation time of any solution is thereby a critical issue. Given the complexity of the problem, the computation time to find an optimal solution either with our MIP model or with other exact approaches is unsuited for real-time applications. Therefore, we introduce a novel heuristic search approach based on the Optimistic Planning (OP) paradigm [25], [23], capable of computing solutions that do not deviate too much from the FCFS solution sequence and that are relatively close to optimal solutions, within an acceptable computational time.

Our approach models the ALP as an environment defined by states, transitions, actions, and costs where:

- each state denoted $x$, is a partition $(I, \bar{I})$ of the set of aircraft, where $\bar{I}$ is the (ordered) set of aircraft that have already landed, and $I$ is the set of aircraft that have not landed yet.

- each action denoted $u$ is an aircraft index $i \in I$ that we decide to land.

- each transition is defined as follows. If we execute action $u=i \in I$ from a given state $x=(I, \bar{I})$, then the system generates the unique next state $x^{\prime}=\left(I^{\prime}, \bar{I}^{\prime}\right)$, where $I^{\prime}=I \backslash\{i\}$, and $\bar{I}^{\prime}=\bar{I} \cup\{i\}$ (aircraft $i$ landed).

- when the environment transits from the state $x$ to the new state $x^{\prime}$ defined above, the estimated value $c$ (cost) of the the new state is defined by

$$
c=f\left(\bar{I}^{\prime}\right)+g\left(I^{\prime}\right),
$$

where $f\left(\bar{I}^{\prime}\right)$ is the delay cost of the (landed) sequence $\bar{I}^{\prime}$, and $g\left(I^{\prime}\right)$ is a function that estimates the lowest cost among all sequences obtained from $I^{\prime}$ that satisfy the CPS constraints. In our numerical experiments, the FCFS rule is chosen as the estimation heuristic $g$, i.e. $m=0$.

Optimistic Planning is the method that incrementally explores this search tree so as to identify an optimal branch as quickly as possible. The algorithm starts from the initial state where the set $\bar{I}$ is empty, and $I=\mathcal{A}$ (all aircraft available to land). At each iteration, its main loop seeks to determine which aircraft to land based on the optimistic evaluation $c$, and it updates $\bar{I}$ by adding this aircraft, until a stopping criteria is met, i.e. all aircraft are landed or a time limit is reached. Only actions that satisfy the operational constraints are available in a given state.

\section{RESULTS AND DISCUSSION}

In this section we report the computational results of the MIP formulation (1)-(12) and of our Optimistic Planning approach. Experiments are run on a personal computer under GNU/Linux operating system, processor Intel(R) Core(TM)

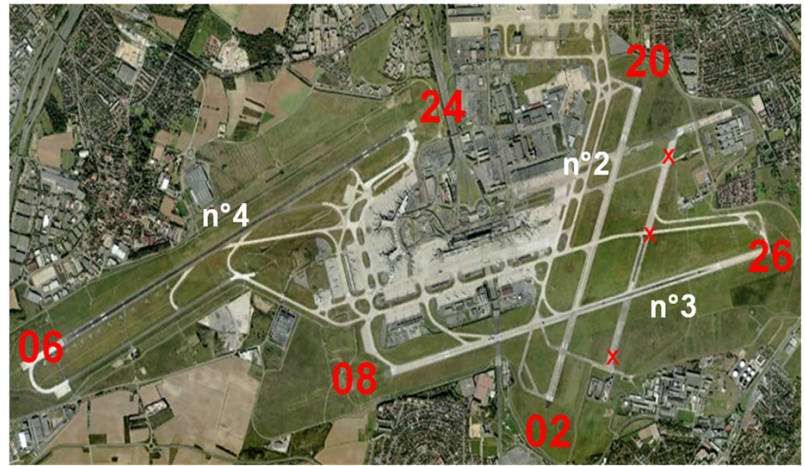

Figure 2: A representation of Orly runways (Source [26])

i7-4700M with 8 GB of RAM. The MIP model was implemented in DoCplex, and solved using IBM CPLEX (version 12.8). Before reporting the computational results, we first present the test instances used in this paper.

\subsection{Test instances}

We use four test instances from a benchmark test-problem set under construction at ENAC, obtained from real traffic in Paris-Orly Airport, that features two runways (06/24 and $08 / 26$ as shown in Figure 2), which are considered independent (runway 02/20 is rarely used for commercial traffic).

These instances are constructed from two traffic days obtained from the OpenSky Network [27]: one in July 2018 containing mostly data about landed aircraft on runway 06/24, and one in April 2019 containing data about landed aircraft on runway 08/26. The four instances come from merging these two traffic days and artificially adding light aircraft to obtain larger and also more congested instances. The four considered instances are available at [28].

Table 3 shows an example of such an instance. The fourth and fifth columns, denoted "sta" and "sta_s", indicate the scheduled time of arrival in HH:MM:SS format and in seconds respectively. The sixth column displays the delay cost per time unit of each aircraft, that we computed following a similar approach to that used in [8].

\subsection{Computational results}

We first report results obtained from implementing our MIP model involving a single runway $(|\mathcal{K}|=1)$, for different values of the maximum position shifting $m=2, \ldots, 6$. Figure 3 illustrates the evolution of the computation time in seconds for each value of $m$ and for a set of 10 test instances of various sizes $|\mathcal{A}|=16,18, \ldots, 30$, obtained by simply considering the first $|\mathcal{A}|$ lines of each of the four instances [28]. We impose a time limit of 1800 seconds (30 minutes) in CPLEX.

Figure 3 exhibits the expected exponential growth of the computing time with the size of the instance, $|\mathcal{A}|$, and with increasing values of $m$, (recall that the ALP is an NP-hard 


\begin{tabular}{|cccccc|}
\hline index & mdl & category & sta & sta_s & delay cost \\
\hline 1 & A332 & Heavy & $07: 00: 00$ & 25200 & 8 \\
2 & - & Light & $07: 00: 00$ & 25200 & 1 \\
3 & B744 & Heavy & $07: 00: 00$ & 25200 & 22 \\
4 & A333 & Heavy & $07: 00: 00$ & 25200 & 8 \\
5 & B744 & Heavy & $07: 00: 00$ & 25200 & 22 \\
6 & B738 & Medium & $07: 10: 00$ & 25800 & 7 \\
7 & A320 & Medium & $07: 15: 00$ & 26100 & 5 \\
8 & B77W & Heavy & $07: 15: 00$ & 26100 & 28 \\
9 & A333 & Heavy & $07: 15: 00$ & 26100 & 8 \\
10 & A320 & Medium & $07: 20: 00$ & 26400 & 5 \\
11 & A320 & Medium & $07: 20: 00$ & 26400 & 5 \\
12 & B738 & Medium & $07: 25: 00$ & 26700 & 7 \\
13 & A318 & Medium & $07: 25: 00$ & 26700 & 3 \\
14 & B744 & Heavy & $07: 30: 00$ & 27000 & 22 \\
15 & - & Light & $07: 30: 00$ & 27000 & 1 \\
16 & A321 & Medium & $07: 30: 00$ & 27000 & 7 \\
17 & A318 & Medium & $07: 30: 00$ & 27000 & 3 \\
18 & A320 & Medium & $07: 35: 00$ & 27300 & 5 \\
19 & A320 & Medium & $07: 35: 00$ & 27300 & 5 \\
20 & A320 & Medium & $07: 35: 00$ & 27300 & 5 \\
21 & A319 & Medium & $07: 35: 00$ & 27300 & 4 \\
22 & A321 & Medium & $07: 40: 00$ & 27600 & 7 \\
\hline
\end{tabular}

Table 3: Examples of an instance features with $|\mathcal{A}|=22$ aircraft.

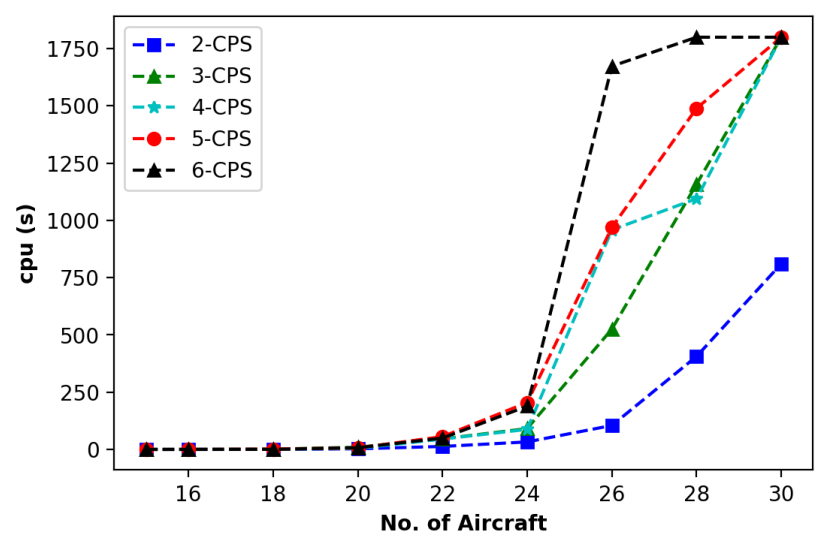

Figure 3: Computational time of the MIP approach for different maximum position shifting values

problem). The saturation effect of 6 -CPS is simply due to our time limit.

Table 4 reports the performance of the MIP model on a test instance of size $|\mathcal{A}|=28$. Throughout Table 4, columns k-CPS represent results for different values of $m$, and the F-CPS (Free CPS) column reports results without CPS constraints. Results are reported in terms of computing time in seconds with a time limit of 1800 seconds, and the percentage improvements with respect to the FCFS sequence that was computed based on the order given by scheduled time of arrivals. The percentage of improvement is computed as:

$$
\text { \%improv }=\frac{C_{\mathrm{FCFS}}-C_{\mathrm{MIP}}}{C_{\mathrm{FCFS}}} \times 100,
$$

where $C_{\mathrm{FCFS}}$ and $C_{\mathrm{MIP}}$ are the costs of the FCFS sequence and that of the MIP solution calculated via CPLEX, respec- tively.

\begin{tabular}{|ccccccc|}
\hline & 2-CPS & 3-CPS & 4-CPS & 5-CPS & 6-CPS & F-CPS \\
\hline cpu (s) & 5 & 66 & 154 & 240 & $\mathbf{1 8 0 0}$ & $\mathbf{1 8 0 0}$ \\
\%improv & 25 & 38 & 50 & 57 & 64 & 64 \\
\hline
\end{tabular}

Table 4: MIP approach performance on a test instance of size $|\mathcal{A}|=28$ for different maximum position shifting values

It can be concluded from Table 4 that significant improvements can be obtained starting from $m=3$, but this requires large computation times, which make it non-adapted to the dynamic nature of our problem, especially since the complexity scales exponentially with the number of aircraft, and since future ATM systems will have to deal with very large ALP instances.

We report the results of the OP approach on the mediumsized instances used in Figure 3, as well as on large instances of sizes $|\mathcal{A}|=30,32, \ldots, 40$ involving a single runway (Table 5), and imposing each time a limited computational time-budget of $\{2,5,15\}$ seconds. For each instance size, we evaluate the approach on different instances of the same size and report the average, minimum and maximum improvement over the FCFS solution.

Table 5 shows the average percentage improvement of the total cost given by Eq. (13) for two values of the maximum position shifting parameter, $m=2$ and 3 . It can be observed that large instances of sizes greater than 30 can benefit from a significant improvement (on average more than $21 \%$ ) for a maximum position shifting of $m=3$, within only 5 seconds.

Finally, Table 6 reports an example of the solution provided by our OP approach on the instance given in Table 3 with $|\mathcal{A}|=22$ aircraft, and imposing a computational budget of 2 seconds. The first column displays the aircraft position in the solution sequence. The "index" columns corresponds

\begin{tabular}{|ccccccc|}
\hline \multicolumn{3}{c}{ Average improvement $(\%)$} & \multicolumn{3}{c|}{ Average improvement $(\%)$} \\
$\mathbf{k}=2$ & \multicolumn{3}{c}{$\mathbf{k}=3$} \\
\hline & $2 s$ & $5 s$ & $15 s$ & $2 s$ & $5 s$ & $15 s$ \\
\hline 18 & 12 & 12 & 12 & 2 & 15 & 15 \\
20 & 18 & 18 & 19 & 10 & 23 & 24 \\
22 & 23 & 22 & 21 & 19 & 32 & 33 \\
24 & 19 & 25 & 26 & 22 & 26 & 31 \\
26 & 14 & 19 & 20 & 11 & 24 & 31 \\
28 & 15 & 16 & 24 & 9 & 23 & 29 \\
30 & 14 & 18 & 24 & 8 & 21 & 36 \\
32 & 14 & 18 & 26 & 2 & 22 & 37 \\
34 & 14 & 12 & 25 & 6 & 23 & 28 \\
36 & 13 & 13 & 25 & 7 & 24 & 24 \\
38 & 12 & 13 & 26 & 8 & 23 & 26 \\
40 & 12 & 13 & 25 & 7 & 22 & 26 \\
\hline \hline $\min$ & 12 & 12 & 12 & 2 & 15 & 15 \\
$\max$ & 23 & 25 & 26 & 22 & 32 & 37 \\
avg & 15 & 16 & 20 & 28 & 23 & 29 \\
\hline
\end{tabular}

Table 5: Algorithm performance details (average improvement) for one runway 


\begin{tabular}{ccccc}
\hline & \multicolumn{2}{c}{ FCFS } & \multicolumn{2}{c}{ OP } \\
\hline Position & index & landing & index & landing \\
\hline 1 & 1 & $7: 00: 00$ & 3 & $7: 00: 00$ \\
2 & 2 & $7: 03: 16$ & 5 & $7: 01: 36$ \\
3 & 3 & $7: 04: 16$ & 4 & $7: 03: 12$ \\
4 & 4 & $7: 05: 52$ & 1 & $7: 04: 48$ \\
5 & 5 & $7: 07: 28$ & 2 & $7: 08: 04$ \\
6 & 6 & $7: 10: 05$ & 6 & $7: 10: 00$ \\
7 & 7 & $7: 15: 00$ & 8 & $7: 15: 00$ \\
8 & 8 & $7: 16: 00$ & 9 & $7: 16: 36$ \\
9 & 9 & $7: 17: 36$ & 7 & $7: 19: 13$ \\
10 & 10 & $7: 20: 13$ & 10 & $7: 20: 22$ \\
11 & 11 & $7: 21: 22$ & 12 & $7: 25: 00$ \\
12 & 12 & $7: 25: 00$ & 13 & $7: 26: 09$ \\
13 & 13 & $7: 26: 09$ & 11 & $7: 27: 18$ \\
14 & 14 & $7: 30: 00$ & 15 & $7: 30: 00$ \\
15 & 15 & $7: 33: 16$ & 14 & $7: 31: 00$ \\
16 & 16 & $7: 34: 25$ & 17 & $7: 33: 37$ \\
17 & 17 & $7: 35: 34$ & 20 & $7: 35: 00$ \\
18 & 18 & $7: 36: 43$ & 21 & $7: 36: 09$ \\
19 & 19 & $7: 37: 52$ & 16 & $7: 37: 18$ \\
20 & 20 & $7: 39: 01$ & 18 & $7: 38: 27$ \\
21 & 21 & $7: 40: 10$ & 19 & $7: 39: 36$ \\
22 & 22 & $7: 41: 20$ & 22 & $7: 40: 45$ \\
\hline
\end{tabular}

Table 6: Examples of solutions provided by FCFS and our optimistic approach

to the aircraft index from Table 3 occupying each position. The "landing" columns report the landing times.

For this example, the percentage improvement of the FCFS sequence is $35 \%$, and it can be deduced from Table 6 that our approach does not only improve the FCFS heuristic in terms of total cost, but also optimizes the utilization of the runway in terms of the makespan, i.e. length of the sequence. Indeed, the last landing in the sequence for the FCFS is 7:41:20 while the last landing with our approach is 7:40:45.

Our computational experiments on the MIP formulation and the heuristic search approach show that the latter is more suited and more promising to solve the ALP with large congested instances, since it can provide good solutions in short computation time.

\section{CONCLUSION}

Runway sequence optimization is an ongoing challenge for researchers and controllers due to the dynamic nature of the problem and to the various operational constraints that must be taken into consideration. In this work, we proposed an exact approach (MIP) to solve the deterministic case of the ALP as well as a novel method based on Optimistic Planning to solve medium and large challenging instances.

Our computational experiments show that computation times for our MIP model (and other exact approaches) are very high for large congested instances, which make them unsuited to the dynamic nature of ALP. With the constrainedposition shifting restrictions, the complexity of the problem can be reduced, but the problem remains untractable for increasing values of the maximum position shifting parameter and the number of aircraft. On the other hand, our proposed heuristic search approach based on optimistic planning is able to find good solutions that significantly improve the FCFS sequence within a limited time budget, making it a promising method for solving the ALP in real time.

Extending our approach to the multiple runway case and taking into consideration uncertainty on the arrival times are future tracks of research.

\section{ACKNOWLEDGMENTS}

The authors would like to thank Serge Roux, from ENAC, for his assistance with technical support.

\section{REFERENCES}

[1] The international air transport association (IATA). [Online]. Available: https://www.iata.org/pressroom/pr/Pages/2017-10-24-01.aspx

[2] A. Odoni and R. De Neufville, Airport systems: Planning, design, and management. McGraw-Hill Professional, 2003.

[3] J. A. Bennell, M. Mesgarpour, and C. N. Potts, "Airport runway scheduling," 4OR, vol. 9, no. 2, 2011.

[4] J. E. Beasley, M. Krishnamoorthy, Y. M. Sharaiha, and D. Abramson, "Transportation science," 4OR, vol. 34, no. 2, pp. 180-197, 2000.

[5] F. Furini, C. A. Persiani, and P. Toth, "Aircraft sequencing problems via a rolling horizon algorithm," in International Symposium on Combinatorial Optimization, pp. 273-284.

[6] D. Briskorn and R. Stolletz, "Aircraft landing problems with aircraft classes," Journal of Scheduling, vol. 17, no. 1, pp. 31-45, 2014.

[7] A. Ghoniem, H. D. Sherali, and H. Baik, "Enhanced models for a mixed arrival-departure aircraft sequencing problem," INFORMS Journal on Computing, vol. 26, no. 3, pp. 514-530, 2014.

[8] F. Furini, M. P. P. Kidd, C. A., and P. Toth, "Improved rolling horizon approaches to the aircraft sequencing problem," Journal of Scheduling, vol. 18 , no. 5 , pp. $435-447,2015$.

[9] A. Faye, "Solving the aircraft landing problem with time discretization approach," European Journal of Operational Research, vol. 242, no. 3, pp. 1028-1038, 2015.

[10] H. Balakrishnan and B. Chandran, "Scheduling aircraft landings under constrained position shifting," in AIAA guidance, navigation, and control conference and exhibit, 2006, p. 6320.

[11] A. Lieder, D. Briskorn, and R. Stolletz, "A dynamic programming approach for the aircraft landing problem with aircraft classes," European Journal of Operational Research, vol. 243, no. 1, pp. 61-69, 2015.

[12] L. Bianco, P. Dell'Olmo, and S. Giordani, "Scheduling models for air traffic control in terminal areas," Journal of Scheduling, vol. 9, no. 3, pp. 223-253, 2006.

[13] A. Salehipour, M. Modarres, and L. M. Naeni, "An efficient hybrid meta-heuristic for aircraft landing problem," Computers \& Operations Research, vol. 40, no. 1, pp. 207-213, 2013.

[14] G. Hancerliogullari, G. Rabadi, A. H. Al-Salem, and M. Kharbeche, "Greedy algorithms and metaheuristics for a multiple runway combined arrival-departure aircraft sequencing problem," Journal of Air Transport Management, no. 32, pp. 39-48, 2013.

[15] A. Rodriguez-Diaz, B. Adenso-Diaz, and P. L. Gonzalez-Torre, "Minimizing deviation from scheduled times in a single mixed-operation runway," Computers \& Operations Research, no. 78, pp. 193-202, 2017.

[16] J. Ma, D. Delahaye, M. Sbihi, P. Scala, and M. A. M. Mota, "Integrated optimization of terminal maneuvering area and airport at the macroscopic level," Transportation Research Part C: Emerging Technologies, no. 98, pp. 338-357, 2019.

[17] B. Soykan and G. Rabadi, "A tabu search algorithm for the multiple runway aircraft scheduling problem," in Heuristics, Metaheuristics and Approximate Methods in Planning and Scheduling, 2016, pp. $165-186$. 
[18] G. Bencheikh, J. Boukachour, A. E. H. Alaoui, and F. E. Khoukhi, "Hybrid method for aircraft landing scheduling based on a job shop formulation," International Journal of Computer Science and Network Security, no. 8, pp. 78-88, 2009.

[19] X. B. Hu and E. A. D. Paolo, "A ripple-spreading genetic algorithm for the aircraft sequencing problem," Evolutionary Computation, no. 19, pp. 77-106, 2011.

[20] G. Bencheikh, J. Boukachour, and A. E. H. Alaoui, "Improved ant colony algorithm to solve the aircraft landing problem," International Journal of Computer Theory and Engineering, no. 3, p. 224, 2009.

[21] Y. Jiang, Z. Xu, X. Xu, Z. Liao, and Y. Luo, "A schedule optimization model on multirunway based on ant colony algorithm," Mathematical Problems in Engineering, 2014.

[22] A. Salehipour, L. Moslemi Naeni, and H. Kazemipoor, "Scheduling aircraft landings by applying a variable neighborhood descent algorithm: Runway-dependent landing time case," Journal of Applied Operational Research, vol. 1, no. 1, pp. 39-49, 2013.

[23] R. Munos, "From bandits to Monte-Carlo tree search: The optimistic principle applied to optimization and planning," Foundations and Trends in Machine Learning, vol. 7, no. 1, pp. 1-129, 2014.

[24] H. Lee and H. Balakrishnan, "A study of tradeoffs in scheduling terminal-area operations," in Proceedings of the IEEE, 2008, pp. 2081-2095.

[25] J. F. Hren and R. Munos, "Optimistic planning of deterministic systems," Foundations and Trends in Machine Learning. Springer, Berlin, Heidelberg, pp. 151-164, 2008.
[26] The paris Orly Airport. [Online]. Available: https://personnel. isae-supaero.fr/emmanuel-rachelson/alp-instances.html

[27] The OpenSky Network. [Online]. Available: https://opensky-network. org

[28] The Aircraft Landing Problem instances. [Online]. Available: https //personnel.isae-supaero.fr/emmanuel-rachelson/alp-instances.html

\section{COPYRIGHT}

\section{Copyright Statement}

The authors confirm that they, and/or their company or institution, hold copyright of all original material included in their paper. They also confirm they have obtained permission, from the copyright holder of any third party material included in their paper, to publish it as part of their paper. The authors grant full permission for the publication and distribution of their paper as part of the EIWAC2019 proceedings or as individual off-prints from the proceedings. 